\title{
Diagnosis of a solitary cardiac metastasis from ocular melanoma
}

\author{
Gabriel T. Chong, BS, Raymond J. Kim, MD, Srivani R. Ambati, MD, Terence Z. Wong, MD, PhD,
} Louis R. Dibernardo, MD, Elmar M. Merkle, MD, Thomas Ryan, MD, and Michael A. Morse, MD, Durham, NC

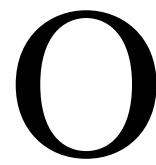
cular melanoma is diagnosed in approximately 6 million people annually. ${ }^{1}$ Although ocular melanoma metastasizes less frequently than cutaneous melanoma ( $75 \%$ vs $96 \%$, respectively), when it does metastasize, it is invariably fatal, usually within 1 year of the onset of symptoms. ${ }^{2}$ Even though the liver is the most common location for metastases from ocular melanoma, it is thought that the heart is another common site that is clinically underdiagnosed.

\section{Clinical Summary}

A 75-year-old white man presented in the fall of 2000 with abdominal pain and shortness of breath. A thorough workup revealed an invasive adenocarcinoma of the cecum. A right hemicolectomy was performed on April 10, 2001, during which a biopsy of a liver lesion identified on computed tomographic (CT) scan was reported to show a metastatic adenocarcinoma. In June 2001, a resection of the left hepatic lobe and placement of an intrahepatic arterial pump were performed to treat what was thought to be metastatic colon cancer. The pathologic results later indicated that the left hepatic lesion was melanoma. Throughout this patient's course, physical examination did not reveal any suggestive skin lesions. A review of the patient's history revealed that ocular melanoma of the right eye had been diagnosed 15 years earlier, and he underwent enucleation at another center. Routine fluorodeoxyglucose positron emission tomography (FDG-PET) and CT scans were unremarkable until March 2005, when the FDG-PET scan revealed a hypermetabolic soft tissue mass in the myocardium of the apex of the left ventricle measuring $24 \times 35 \mathrm{~mm}$ (Figure 1, A, axial, and $B$, coronal). A subsequent contrast-enhanced CT (Figure 1, $A$ ), echocardiogram (Figure 1, $C$ ), and cardiac magnetic resonance imaging (MRI) (Figure 1,D) corroborated this finding.

Cardiac surgery with cardiopulmonary bypass was performed during which an obvious melanotic mass emanating from just to the left of the left anterior descending artery and approximately 2 $\mathrm{cm}$ from the true apex of the heart was found. A sharp dissection was performed with all margins grossly negative. The myocardium resected included parts of the septum, the left ventricle, and the right ventricle. The mass was diagnosed as a cardiac metastasis from ocular melanoma (Figure 2, $A$ and $B$ ).

\footnotetext{
From Duke University Medical Center, Durham, NC.

Funded by Howard Hughes Medical Institute Research Training Fellowships for Medical Students (G.T.C.).

Received for publication July 22, 2005; accepted for publication July 26, 2005 .

Address for reprints: Michael Morse, MD, Box 3233, Duke University Medical Center, Durham, NC 27710 (E-mail: michael.morse@duke.edu).

J Thorac Cardiovasc Surg 2005;130:1727-8

$0022-5223 / \$ 30.00$

Copyright (C) 2005 by The American Association for Thoracic Surgery doi:10.1016/j.jtcvs.2005.07.058
}

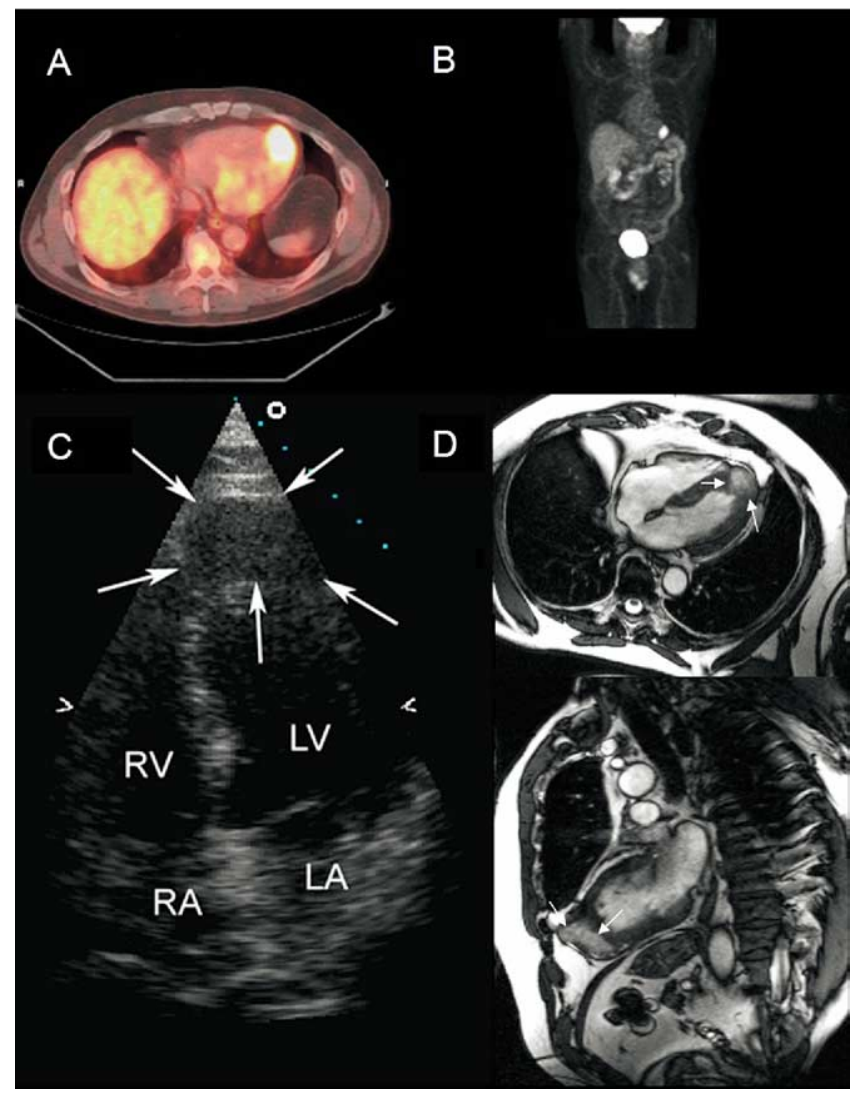

Figure 1. A, Combined axial contrast-enhanced computed tomography (CT) and corresponding fluorodeoxyglucose positron emission tomography (FDG-PET) image shows a $3.2 \mathrm{~cm} \times 3.1 \mathrm{~cm}$ mass in the region of the cardiac apex, which is isodense to muscle tissue during the arterial contrast phase. This mass involves the left ventricle and cardiac septum. B, FDG-PET in March 2005 (B: coronal) revealed a new hypermetabolic soft tissue mass in the myocardium of the apex of the left ventricle measuring $24 \times 35 \mathrm{~mm}$. There was no other evidence of metabolically active disease. C, Apical 4-chamber echocardiogram revealed an apparent extracardiac mass (arrows) adjacent to the LV apex. The dimensions of the mass were $4.3 \times 2.6 \mathrm{~cm}$. D, Cardiac magnetic resonance imaging (MRI) showing a mass measuring $4 \times 2.5 \times 2 \mathrm{~cm}$, which is described as heterogenous but overall bright in image intensity on true-FISP imaging (fast imaging with steady-state precession) before contrast administration, and it did not appear to involve the pericardium. Delayed enhancement imaging demonstrated hyperenhancement of the mass, and perfusion imaging revealed that the mass had a very short $\mathrm{TI}$ even before administration of gadolinium contrast, suggestive of metastatic melanoma. $L A$, Left atrium; $R A$, right atrium; $R V$, right ventricle; $L V$, left ventricle. 


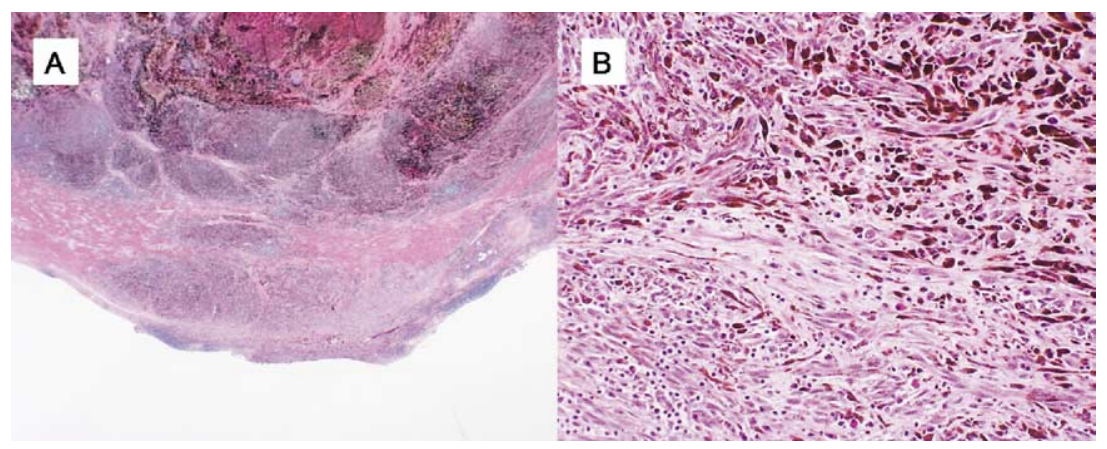

Figure 2. A, Hematoxylin-eosin stain, 2.5x: Resected specimen: $25 \mathrm{~g}, 5.4 \times 3.5 \times 3.3 \mathrm{~cm}$ fragment of tan muscle tissue. Sectioning revealed a $4.9 \times 3.2 \times 2.2 \mathrm{~cm}$ soft, black mass throughout the muscle tissue and extending to the epicardium. B, Hematoxylin-eosin stain, $50 \times$ : Microscopically, large amounts of pigment present throughout the tumor; nuclei are large and pleomorphic with large areas of tumor necrosis.

\section{Discussion}

When ocular melanoma metastasizes, liver involvement is most frequent $(>90 \%)$ followed by lung, skin, bone, thyroid, breast, ovary, adrenal gland, lungs, subcutaneous tissue, lymph nodes, and the central nervous system. ${ }^{1,3,4}$ In terms of cardiac metastases, early reports suggested that such metastases from ocular melanoma are extremely uncommon. ${ }^{3}$ A later report by Mäkitie and Kivelä ${ }^{5}$ suggested that although cardiac metastases from ocular melanoma are indeed clinically underdiagnosed, autopsy reviews indicated that cardiac metastases from ocular melanoma are not uncommon, with $19 \%$ to $24 \%$ of patients who die of disseminated ocular melanoma having cardiac metastasis at autopsy. Typically, these cardiac metastases involve the pericardium and myocardium, and only rarely involve the endocardium or the valves. ${ }^{4}$ The location of this patient's cardiac mass in the left side of the heart is notable because left-sided cardiac involvement is less common. ${ }^{4}$ Melanoma is thought to reach the heart most often through hematogenous spread, as in the case of our patient. ${ }^{4}$ Removal of a cardiac metastasis carries with it the risk of myocardial rupture, as occurred in the case reported by Rosario and colleagues. ${ }^{4}$ In that report, the mass measured $6.2 \times 3.2 \mathrm{~cm}$ compared with a $4.9 \times 3.2$ $\times 2.2 \mathrm{~cm}$ mass that was successfully removed in our patient. ${ }^{4}$

Cardiac metastases from melanomas are generally diagnosed by echocardiography. ${ }^{6}$ Newer detection modalities include magnetic resonance imaging, CT, and PET, which all have established use in detecting distant metastases in patients with melanoma. PET is a helpful, noninvasive imaging technique that is being used today for staging of melanoma and has potential for use in accessing response to treatment. In a study performed in 1998, Holder and coworkers ${ }^{7}$ reported that PET had an overall sensitivity and specificity of $94.2 \%$ and $83.3 \%$, respectively, in contrast with $55.3 \%$ and $84.4 \%$, respectively, for CT in the detection of metastatic disease in melanoma, especially in the liver, soft tissues, and lymph nodes. ${ }^{8}$

\section{Conclusions}

On the basis of a review of the literature and this distinct case, we suggest a vigilant approach to screening patients with a melanoma diagnosis. Diagnosis of cardiac metastasis is especially difficult because patients are often clinically asymptomatic when treatment would be most effective. ${ }^{5}$ Many of them are generally older and have concurrent heart disease, which may obscure clinical and electrocardiogram findings that otherwise would indicate a cardiac metastasis. ${ }^{5}$ Taking advantage of presently available screening modalities (echocardiogram, CT, cardiac MRI, and PET) may lead to earlier diagnosis of metastases, particularly cardiac, leading to more positive outcomes.

\section{References}

1. Damato B. Developments in the management of uveal melanoma. Clin Experiment Ophthalmol. 2004;32:639-47.

2. Bell DJ, Wilson MW. Choroidal melanoma: natural history and management options. Cancer Control. 2004;11:296-303.

3. Ruiz RS, El Harazi S, Albert DM, Bryar PJ. Cardiac metastasis of choroidal melanoma. Arch Ophthalmol. 1999;117:1558-9.

4. Rosario RT, DiMaio DJ, Lapham RL, Sweeney M, Smalling R, Barasch E. Metastatic ocular melanoma to the left ventricle inducing nearsyncope attacks in an 84-year-old woman. Chest. 2000;118:551-3.

5. Mäkitie T, Kivelä T. Cardiac metastasis from uveal melanoma. Chest. 2000;120:2115-6.

6. Bossert T, van Son JAM, Autschbach R, Mohr FW. Resection of a right atrial metastatic melanoma with unknown origin of primary tumor. Eur J Cardiothorac Surg. 1999;15:373-5.

7. Holder WD Jr, White RL Jr, Zuger JH, Easton EJ Jr, Greene FL. Effectiveness of positron emission tomography for the detection of melanoma metastases. Ann Surg. 1998;227:764-9; discussion 76971.

8. Friedman KP, Wahl RL. Clinical use of positron emission tomography in the management of cutaneous melanoma. Semin Nucl Med. 2004;34: $242-53$. 